Results The virtual induction runs live over two days, with homework, in the form of the filmed consultations that can be accessed at any time. The first day has been completed, delivering training to around 20 new trainees. The feedback we have received from both trainees and training programme directors has been overwhelmingly positive.

Discussion Given the ongoing impact of the coronavirus pandemic, creative ways of delivering training are flourishing. We have created a bank of videos and presentations, as well as a template for future induction sessions, ensuring a basic level of equity between trainees at different genetics centres.

Conclusion Virtual induction tools are a valuable and vital addition to new trainee induction to ensure equity of training, both during the current pandemic and beyond. This approach would work very well on a national level for other small specialties; or on a regional or local level for larger specialties.

\section{AUDIT OF POST-HSCT ENDOCRINE MONITORING FOR METABOLIC PATIENTS}

Nazreen Banu Kamarus Jaman, James Davison. Great Ormond Street Hospital

\subsection{6/archdischild-2020-gosh.78}

Background We have completed an audit to review our current routine endocrine surveillance of a cohort of metabolic patients who have had a haematopoietic stem cell transplant (HSCT) and ensure relevant investigations are obtained, and appropriate referral to endocrine teams are made.

Aim Describe long term outcome of HSCT in children at risk of long term endocrine problems.

To review whether post-HSCT metabolic patients are having the required endocrine complication-surveillance investigations undertaken, and if not to correct the problem

Methods

1. All patients with confirmed diagnosis of MPS $1 \mathrm{H}$ from a single tertiary centre were included.

2. 3 MPS2 \& 1 Alpha Mannosidosis included.

3. A retrospective case notes review was undertaken to assess the following outcome measures:

4. Post HSCT growth parameters

5. Thyroid function tests

6. Sex hormone levels

7. Specific endocrine issues.

Discussion

1. We have noted among 31 patients, 16 patients were referred to endocrine team.

2. 7 patients are referred at or after the 10 years of age whilst the rest of the 9 patients had endocrine assessment at the mean age of 2-9 years.

3. The endocrine referral done before $10 \mathrm{yrs}$ of age was because of specific endocrine problems, not as a surveillance.

4. Most of the patients had the growth parameters checked however it was not particularly focussed or had set points for regular monitoring. We have learnt that only $10 \%$ of patients had sex hormone levels checked at the adolescent age when the expected endocrine issues are anticipated.

Conclusion Overall, we highlight that proper endocrine referral at the age of 10 years is required for the post HSCT MPS patients.

\section{DOES A REDUCED FORCED VITAL CAPACITY COMPARE WITH ABNORMAL SLEEP STUDY RESULTS?}

Ben Griffiths, Sreeja Kiran, Joanne Martins, Stephanie Brotherston, Matthew Davies, Aidan Laverty. Great Ormond Street Hospital

\subsection{6/archdischild-2020-gosh.79}

Introduction Respiratory deterioration impacts morbidity and mortality in patients with Neuromuscular disorders (NMD). Respiratory involvement will vary by disorder; patients with Duchenne muscular dystrophy (DMD) and Spinal muscular atrophy (SMA) often displaying deterioration with disease progression. Such patients may present with sleep-related breathing disorders (SRBD) which can progress to hypoventilation. Spirometry, including forced vital capacity (FVC) measurements, monitor respiratory muscle strength and sleep studies identify SRBD to determine when non-invasive ventilation is required. Assessing when to refer for a sleep study is therefore clinically important. Previous research suggests the use of FVC $<60 \%$ of predicted normal (FVCLT60\%) may indicate an abnormal sleep study.

Aim To assess if patients with FVCLT60\% had an abnormal baseline sleep study.

Method A retrospective study of patients who attended for spirometry between April 2018 - April 2020 with FVCLT60\% and a baseline cardiorespiratory sleep study within 3 months of spirometry. Patients were excluded if using night time respiratory support. Apnoea-Hypopnoea Index (AHI) was used to classify SRBD. Fifty-four patients were identified and results reported as mean $( \pm S D)$.

Results Demographics: Age $14.05( \pm 2.8)$ years, 40 male (74\%), 30 DMD (56\%). Twenty-Two (40.7\%) had moderate or severe SRBD (AHI $\geq 5$ events/hour), with a mean $\mathrm{FVC} \%$ predicted of $37.6 \%( \pm 12.0 \%)$. Those with mild or no SRBD (AHI $<5$ events/hour) showed mean FVC of $44.5 \%( \pm 9.7 \%)$. Conclusion Our study showed $59 \%$ of patients who had FVCLT60\% had mild or no SRBD. In our patient group, the use of FVCLT60\% may not be appropriate for sleep study referral. A lower FVC threshold may be more appropriate in identifying patients who require treatment for SRBD, but further research is required.

\section{VIDEO FLUOROSCOPY SWALLOW SERVICE; AN ALLIED HEALTH PROFESSIONAL COLLABORATION}

${ }^{1}$ Jessica Eaton, ${ }^{2}$ Alex Stewart, ${ }^{1}$ Rebekah Bennison, ${ }^{3}$ lan C Simcock. ${ }^{1}$ Great Ormond Street Hospital; ${ }^{2}$ Great Ormond Street Hospital and University College London; ${ }^{3}$ Great Ormond Street Hospital/Institute of Child Health NIHR BRC and University College London

\subsection{6/archdischild-2020-gosh.80}

Allied Health Professionals (AHPs) form the third largest clinical workforce in health and social care in England (NHS England, 2017). Traditionally, the AHP workforce has provided high quality care to patients across a wide range of care pathways and in a variety of settings. In recent times, efforts to modernise the health service have included a drive towards a more flexible workforce and a reconsideration of the roles of different members of the clinical team. This transition has facilitated the formation of multidisciplinary teams involving a range of AHPs providing a key opportunity to improve access 
to care, reduce waiting times and NHS costs (Department of Health, 2014).

One example of this collaboration is the Video Fluoroscopy Swallow Service provided by Radiographers and Speech and Language Therapists (SLT) at Great Ormond Street Hospital. The service provides accurate, low dose x-ray screening for the assessment of swallow physiology in children. Results are used to inform multi-disciplinary management of eating and drinking difficulties, including aspiration risk. We outline the service and explain how this method of collaborative working harnesses the clinical expertise of both professions, providing a high-quality service which contributes to the improvement of patient outcomes and experience.

Learning outcomes from this service include the importance of having clearly defined AHP roles, and patient suitability criteria as well as excellent communication between the whole multi-disciplinary team. The opportunity for extended scope practice provides staff with job satisfaction and skill development. This Video Fluoroscopy swallow study model highlights how AHP's working collaboratively can make a vital contribution to efficient use of clinical spaces, reducing costs and waiting lists while providing high quality care for children at Great Ormond Street Hospital.

It is essential that such collaborations at Great Ormond Street Hospital are celebrated and actively encouraged enabling service improvements.

\section{CONTROLLED DRUGS STORAGE AND IMPROVEMENT}

${ }^{1}$ Andrew Pearson, ${ }^{2}$ Hong Thoong, ${ }^{2}$ Rasha Shamsah, ${ }^{2}$ Ka Yu Yung. ${ }^{1}$ Great Ormond Street Hospital; ${ }^{2} \mathrm{GOSH}$

\subsection{6/archdischild-2020-gosh.81}

Learning from a Serious Incident in 2018 highlighted the importance of the documentation of controlled drugs. A detailed audit was conducted in July 2019. The main areas for improvement across the trust were around documentation in the $\mathrm{CD}$ order book and $\mathrm{CD}$ register. An action plan was agreed between nursing education and pharmacy departments to support best practice. This included the development of digestible best practice guidance to be displayed in medicine storage rooms, revision of policy and education roll out which took take place in September 2019 Changes have been made and through the audit process we have improved our standards.

$\%$ of all standards met

Jul 2019 80\%

December 2019 85\%

August 2020 90\%

Feeding back audit results quickly and visually has been useful. Thankyou to all pharmacists and ward staff who have supported this work. We have made changes and reduced risk in this area.

\section{EVALUATION OF THE MEDICINES MATTER NEWSLETTER IN GOSH}

Ka Yu Yung. Great Ormond Street Hospital

10.1136/archdischild-2020-gosh.82
Medicines form the largest group of interventions used in children and young people. It also accounts for the highest number of clinical errors. A newsletter 'Medicines Matter' designed to support good use of medicines within the Trust was introduced in October 2019 and published monthly by Pharmacy. The newsletter, intended to be read by all healthcare professionals, highlights issues and provides updates on all aspects of medicines management. The contents of the newsletter includes incident examples, along with tips in mitigation, new drugs approved by the Drugs \& Therapeutic Committee, new resources, MHRA drug safety update, drug shortages, practice and governance issues.

A feedback survey was conducted to evaluate its' effectiveness as a communication forum as well as staff's views of it. A survey with 9 questions was circulated with the July 2020 edition and answers were collected until September 2020. Sixteen staff members completed the survey where nursing staff dominated (69\%), followed by allied health professionals (25\%) and medical staff (6\%). $80 \%$ of participants read every or most issues with the remaining reading some issues. All participants rated the newsletter as good or very good and useful or very useful where at least $60 \%$ of the respondents were interested in each of the topics within the newsletter. The content was found to be engaging (53\%), educational (80\%), informative (100\%), fun-to-read (33\%) and a quick reference to changes that are happening $(7 \%)$. A larger percentage $(60 \%)$ of participants did not know where to find the newsletter and a suggestion were made to include some medication-related success stories and addition of hyperlinks to new resources available. One participant found the layout and format slightly disorientating but the overall response was positive. The feedback suggests that the newsletter provides a good platform to deliver up-to-date information and key communication across the organisation.

\section{A MULTIVARIABLE COMPOSITE OUTCOME TO DEFINE DISEASE SEVERITY IN CHILDREN WITH CYSTIC FIBROSIS}

${ }^{1}$ Nicole Filipow, ${ }^{1}$ Gwyneth Davies, ${ }^{1}$ Eleanor Main, ${ }^{2}$ Sanja Stanojevic. ${ }^{1}$ Great Ormond Street Hospital and University College London/ICH BRC; ${ }^{2}$ Dalhousie University

\subsection{6/archdischild-2020-gosh.83}

Introduction Improvements in cystic fibrosis (CF) care have resulted in improved outcomes, specifically many children maintain lung function in the normal range. Nonetheless there are children with poor outcomes, and there is a need for a more comprehensive multi-factorial measure that summarises the overall health status in this new era of CF care.

Objective To define phenotypically distinct clusters of pediatric CF patients that are linked to different health outcomes by using basic machine learning algorithms.

Method Data from the Toronto CF Clinical Database were used to define phenotypic clusters based on a broad variety of patient-descriptive variables. A Partitioning Around Medoids (PAM) clustering method was iteratively carried out on different combinations of the variables until a maximum distinction between outcome measures could be identified, which included time to recurrent event analyses for both pulmonary exacerbations and hospital admissions. The results were validated in GOSH CF clinical data housed within the GOSH-DRIVE DRE. 